\title{
COMP-angiopoietin-1 promotes wound healing through enhanced angiogenesis, lymphangiogenesis, and blood flow in a diabetic mouse model
}

\author{
Chung-Hyun Cho*, Hoon-Ki Sung*, Kyung-Tae Kim*, Hyae Gyeong Cheon ${ }^{\dagger}$, Goo Taeg Oh, Hyo Jeong Hong\$, \\ Ook-Joon Yoo*, and Gou Young Koh*ף

\begin{abstract}
*Biomedical Research Center and Department of Biological Sciences, Korea Advanced Institute of Science and Technology, Daejeon 305-701, Korea; ${ }^{\dagger}$ Division of Medicinal Science, Korea Research Institute of Chemical Technology, Daejeon 305-600, Korea; ${ }^{\ddagger}$ Division of Molecular Life Sciences, Ewha Woman's University, Seoul 120-750, Korea; and §Antibody Engineering Research Unit, Korea Research Institute of Bioscience and Biotechnology, Daejeon 305-600, Korea
\end{abstract}

Edited by George D. Yancopoulos, Regeneron Pharmaceuticals, Inc., Tarrytown, NY, and approved February 3, 2006 (received for review July 26, 2005)

\begin{abstract}
Microvascular dysfunction is a major cause of impaired wound healing seen in diabetic patients. Therefore, reestablishment of structural and functional microvasculature could be beneficial to promote wound healing in these patients. Angiopoietin-1 (Ang1) is a specific growth factor functioning to generate a stable and functional vasculature through the Tie 2 and Tie1 receptors. Here we determined the effectiveness of cartilage oligomeric matrix protein (COMP)-Ang1, a soluble, stable, and potent form of Ang1, on promotion of healing in cutaneous wounds of diabetic mice. An excisional full-thickness wound was made in the dorsal side of the tail of diabetic $(d b / d b)$ mice, and mice were then treated systemically with adenovirus (Ade) encoding COMP-Ang1 or with control virus encoding $\beta$-gal (Ade- $\beta$-gal) or treated topically with recombinant COMP-Ang1 protein or BSA. Time course observations revealed that mice treated with Ade-COMP-Ang1 or COMP-Ang1 protein showed accelerated wound closure and epidermal and dermal regeneration, enhanced angiogenesis and lymphangiogenesis, and higher blood flow in the wound region compared with mice treated with control virus or BSA. COMP-Ang1 promotion of wound closure and angiogenesis was not dependent on endothelial nitric oxide synthase or inducible nitric oxide synthase alone. Taken together, these findings indicate that COMP-Ang1 can promote wound healing in diabetes through enhanced angiogenesis, lymphangiogenesis, and blood flow.
\end{abstract}

diabetes | growth factor | cutaneous wound | therapeutic protein nitric oxide

$\mathbf{H}$ ealing of an adult cutaneous (skin) wound is a complex process integrating activities of different tissues and cell lineages (1). How contributing cell types behave during proliferation, migration, matrix synthesis, and contraction, as well as growth factors and matrix signals present at a wound site in normal and pathologic conditions, have been extensively investigated. Of these contributions, angiogenesis and lymphangiogenesis are crucial to the wound-healing process $(2,3)$. Signals mediated by VEGF and angiopoietin have been implicated in control and regulation of angiogenesis and lymphangiogenesis $(4,5)$.

Delayed skin wound healing is a serious complication in diabetes and is caused primarily by microangiopathy and peripheral neuropathy accompanied by impaired cutaneous blood flow, hypoxia, accelerated inflammation, edema, and endothelial-neural dysfunction (6-9). Moreover, expression of VEGF-A and Tie2, the angiopoietin-1 (Ang1) receptor, is markedly reduced in wounds in diabetes $(10,11)$. Therefore, restoring structural and functional microvasculature by supplementary delivery of VEGF-A or Ang1 could be beneficial to promote wound healing in diabetes. In fact, recent reports $(12,13)$ indicate that topical application of VEGF-A promotes cutaneous wound healing through increased angiogenesis and by mobilizing and recruiting bone marrow-derived cells. How- ever, exogenous VEGF-A often results in leaky, inflamed, and malformed vessels, greatly compromising its therapeutic utility (12-14). In comparison, Ang1 is a specific growth factor functioning to generate a stable and functional vasculature through Tie 2 and Tie1 receptors (14-18). In addition to Ang1 activating Tie2 and Tie1 signaling, recent reports $(19,20)$ show that Ang1 directly interacts with myocytes, endothelial cells, and fibroblasts through integrins to mediate survival, cell adhesion, and migration. We have recently developed a soluble, stable, and potent Ang1 recombinant chimera, COMP-Ang1 (21). To do so, we replaced the aminoterminal of Ang1 with the short coiled-coil domain of cartilage oligomeric matrix protein (COMP). COMP-Ang1 is more potent than native Ang1 in phosphorylating the Tie2 receptor and signaling through Akt in primary cultured endothelial cells (21). Furthermore, long-term and sustained treatment with COMP-Ang1 could produce long-lasting and stable vascular enlargement and increased blood flow (17).

In the present study, we determined the effectiveness of COMPAng1 in promoting healing of cutaneous wounds of normal and diabetic mice. In addition, because Ang1-induced angiogenesis appears to require generation of nitric oxide by activated endothelial nitric oxide synthase (eNOS) of the endothelium (22), we asked whether eNOS or inducible nitric oxide synthase (iNOS) participated in COMP-Ang1-induced accelerated wound healing by using eNOS $(-/-)$ and $i \operatorname{NOS}(-/-)$ mice. Our results indicate that COMP-Ang1 can promote wound healing in normal and diabetic mice accompanied by enhanced angiogenesis, lymphangiogenesis, and blood flow. COMP-Ang1-induced promotion of wound closure and angiogenesis was not dependent on eNOS or iNOS alone.

\section{Results and Discussion}

COMP-Ang1 Promotes Angiogenesis, Lymphangiogenesis, and Wound Healing in Ear Skin of Normal Mice. To investigate wound healing in vivo, we made ear-punch injuries in mice treated systemically with $1 \times 10^{9}$ plaque-forming units (pfu) of adenovirus (Ade) encoding COMP-Ang1 or with $1 \times 10^{9}$ pfu of Ade- $\beta$-gal, which are hereafter referred to as COMP-Ang1 or control, unless otherwise specified. At 3 and 7 days after injury, larger numbers of delicate vessels were observed around the margin of punched-hole injuries in mice treated with COMP-Ang1 compared with control-treated mice

Conflict of interest statement: No conflicts declared.

This paper was submitted directly (Track II) to the PNAS office.

Abbreviations: Ade, adenovirus; Ang1, angiopoietin-1; COMP, cartilage oligomeric matrix protein; eNOS, endothelial nitric oxide synthase; iNOS, inducible nitric oxide synthase; LYVE-1, lymph vessel endothelial hyaluronan receptor-1; PECAM-1, platelet-endothelial cell adhesion molecule-1; pfu, plaque-forming unit.

"To whom correspondence should be addressed at: Biomedical Research Center, Korea Advanced Institute of Science and Technology, 373-1, Guseong-dong, Daejeon 305-701, Republic of Korea. E-mail: gykoh@kaist.ac.kr.

C 2006 by The National Academy of Sciences of the USA 

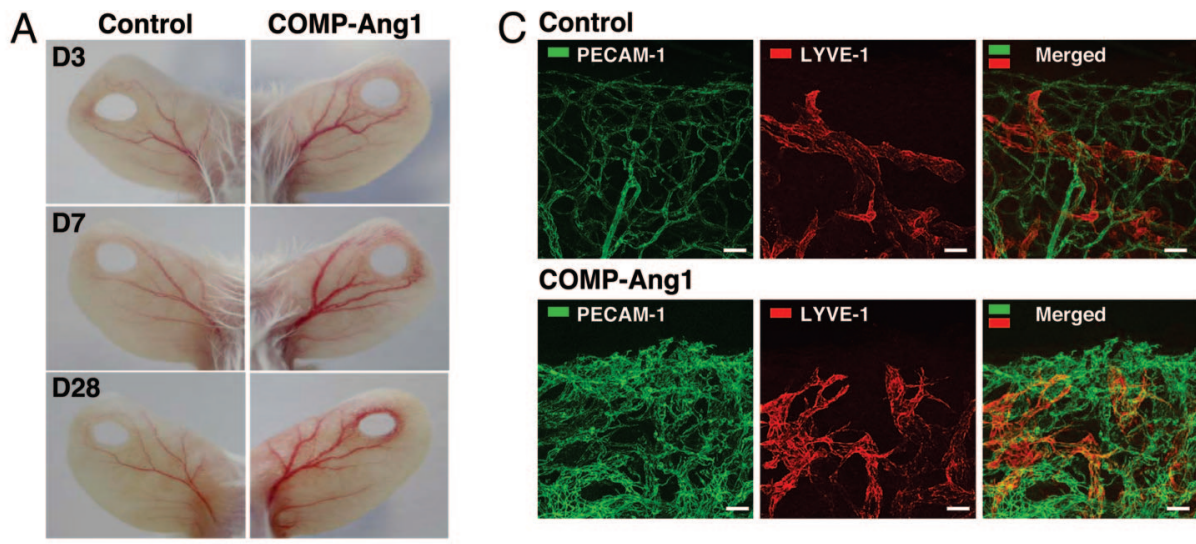

COMP-Ang1
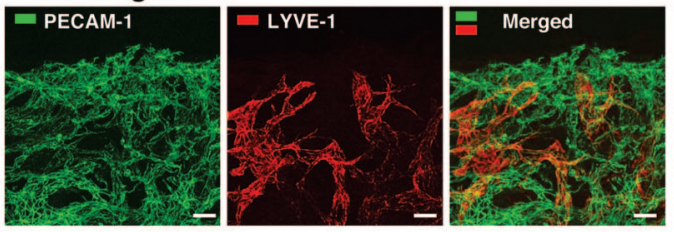

B

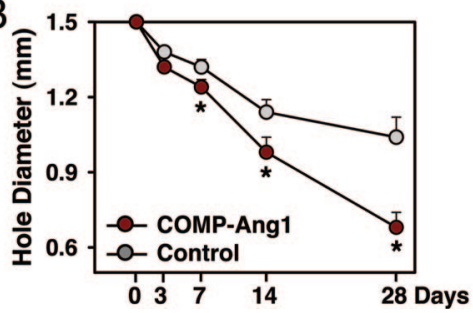

D

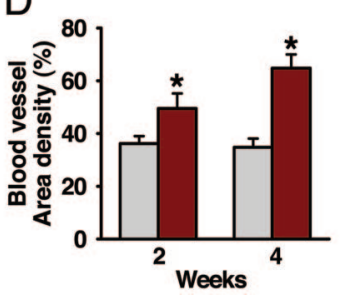

$\mathrm{E}$

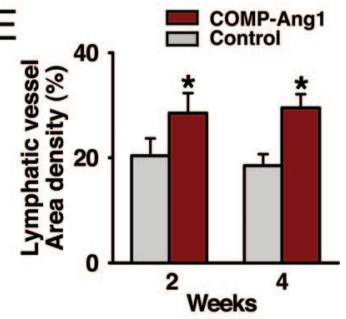

Fig. 1. COMP-Ang1 promotes angiogenesis, lymphangiogenesis, and wound healing in ear skin. FVB/ $\mathrm{N}$ mice were treated with $1 \times 10^{9}$ pfu of Ade- $\beta$-gal (Control) or Ade-COMP-Ang1 (COMP-Ang1) virus, and a closed punched-hole injury was made in the ear. At the indicated days (D) later, ears were photographed $(A)$ and hole diameter was measured $(B)$. (C) Blood and lymphatic vessels at healing margins were visualized with PECAM-1 (green) and LYVE-1 (red) immunostaining, respectively, 28 days after treatment. (Scale bars, $50 \mu \mathrm{m}$.) Area densities of blood $(D)$ and lymphatic $(E)$ vessels were measured. Mice treated with COMP-Ang1 show improved wound healing with enhanced densities of blood and lymphatic vessels in ear skin. All circles shown in $B$ and all bars shown in $D$ and $E$ represent mean \pm SD from four mice. *, $P<$ 0.01 versus control at each time point.
(Fig. 1A). Moreover, blood vessels on the ipsilateral side of the ear, leading from the base to the injury site, were notably enlarged in mice treated with COMP-Ang1 compared with control-treated mice (Fig. 1A). These conditions persisted for up to 28 days after treatment (Fig. 1 $A$ ). The extent of wound healing was inferred from the closure of holes relative to the initial wound diameter. In COMP-Ang1 $(n=5)$ versus controls $(n=5)$, that hole diameter was $1.74 \mathrm{~mm}$ versus $1.82 \mathrm{~mm}$ on day $7, P<0.01 ; 1.48 \mathrm{~mm}$ versus $1.64 \mathrm{~mm}$ on day $14, P<0.01$; and $1.18 \mathrm{~mm}$ versus $1.54 \mathrm{~mm}$ on day $28, P<0.01$ (Fig. $1 B$ ). Immunofluorescence using the blood vessel endothelial cell marker, platelet-endothelial cell adhesion molecule-1 (PECAM-1), and the lymphatic vessel endothelial cell marker, lymph vessel endothelial hyaluronan receptor-1 (LYVE-1), in whole mounted tissues of ear skin revealed that COMP-Ang1treated mice had more enlarged and denser blood and lymphatic vessels in the marginal region of the healing region than did control-treated mice (Fig. 1C). Overall blood vessel densities (PECAM-1 immunopositive areas/total areas) in the healing ear margin of COMP-Ang1-treated mice $(n=4)$ were 1.37 -fold $(P<$ $0.01)$ and 1.86 -fold $(P<0.01)$ greater than that seen in control mice $(n=4) 2$ and 4 weeks, respectively, after treatment (Fig. 1D). Moreover, overall lymphatic vessel densities (LYVE-1 immunopositive areas/total areas) in the healing ear margin dermis of COMP-Ang1-treated mice $(n=4)$ were 1.40 -fold $(P<0.01)$ and 1.59 -fold $(P<0.01)$ greater than those observed in control mice $(n=4)$ at 2 and 4 weeks, respectively, after treatment (Fig. $1 C$ and $D$ ). These observations suggest that more efficient wound healing in the ear of mice treated with COMP-Ang1 resulted from enhanced angiogenesis and lymphangiogenesis in the injured area.

COMP-Ang1 Accelerates Wound Healing and Promotes Angiogenesis, Lymphangiogenesis, and Blood Flow in Tail Skin of Diabetic Mice. The above results led us to investigate the effect of COMP-Ang1 on delayed cutaneous wound healing seen in diabetes, which is mainly caused by microangiopathy (6-9). To do so, we made excisional full thickness wounds in the dorsal side of the tail, where contraction is minimal (23), of diabetic C57BLKS/J-m +/ $+\operatorname{Lepr}^{d b}(\mathrm{db} / \mathrm{db})$ mice (24), whose phenotype resembles that of human adult-onset type II diabetes mellitus. COMP-Ang1 or control was administered intra- venously $12 \mathrm{~h}$ after wounding. In Ade-COMP-Ang1-treated diabetic $(d b / d b)$ mice, circulating COMP-Ang1 levels increased as early as $12 \mathrm{~h}(355 \pm 98 \mathrm{ng} / \mathrm{ml})$, peaked at 1 week $(3,221 \pm 365$ $\mathrm{ng} / \mathrm{ml})$, declined gradually, and returned to control levels 6 weeks after Ad-COMP-Ang1 administration (Fig. 6, which is published as supporting information on the PNAS web site), similar to previous findings in Ade-COMP-Ang1-treated FVB/N mice (17). Time course observations indicated that mice treated with COMP-Ang1 showed accelerated wound closure compared with mice treated with control. Wound closure was measured as the area of epidermal closure $\left(\mathrm{mm}^{2}\right)$ from the initial wound after treatment. Values for COMP-Ang1 $(n=5)$ versus control $(n=5)$ mice were $9.3 \mathrm{~mm}^{2}$ versus $3.7 \mathrm{~mm}^{2}$ at 2 weeks, $P<0.05 ; 20.7 \mathrm{~mm}^{2}$ versus $10.1 \mathrm{~mm}^{2}$ at 4 weeks, $P<0.01$; and $28.6 \mathrm{~mm}^{2}$ versus $16.1 \mathrm{~mm}^{2}$ at 8 weeks, $P<$ 0.01 (Fig. $2 A$ and $B$ ). Histological analysis (Table 1, which is published as supporting information on the PNAS web site) over time indicated that mice treated with COMP-Ang1 displayed accelerated epidermal and dermal regeneration, accelerated formation and deformation of granulation tissue reflected by thickness of $\alpha$-smooth muscle actin-positive fibroblast cell layers, and thinner epidermal thickness compared with mice treated with control virus (Fig. $2 C-F$ ). In addition, overall blood vessel densities (PECAM1-immunopositive areas/total areas) in the regenerated dermis of COMP-Ang1-treated mice $(n=5)$ were 1.52 -fold $(P<0.01)$ and 1.77-fold $(P<0.01)$ greater than observations of control mice $(n=$ 5) 2 and 4 weeks, respectively, after treatment (Fig. $3 A$ and $B$ ). Moreover, overall lymphatic vessel densities (LYVE-1-immunopositive areas/total areas) in the regenerated dermis of COMP-Ang1treated mice $(n=5)$ were 2.06-fold $(P<0.01)$ and 2.01-fold $(P<$ $0.01)$ greater than those observed in control mice $(n=5) 2$ and 4 weeks, respectively, after treatment (Fig. $3 C$ and $D$ ). Thus, mice treated with COMP-Ang1 showed enhanced angiogenesis and lymphangiogenesis with enlarged blood and lymphatic vessels in the regenerated dermis compared with mice treated with control. By using a laser Doppler flowmeter (Transonic Systems, Ithaca, NY), tissue blood flow in four regions of the wound area on the dorsal side of the tail was measured (Fig. $3 E$ ). Blood flow rates $(\mathrm{ml} / \mathrm{min}$ per $100 \mathrm{~g}$ of tissue) in four regions of wounded areas of COMPAng1-treated mice were uniformly greater than those in controltreated mice 2 and 4 weeks after treatment (Fig. $3 E$ and $F$ ). Overall 

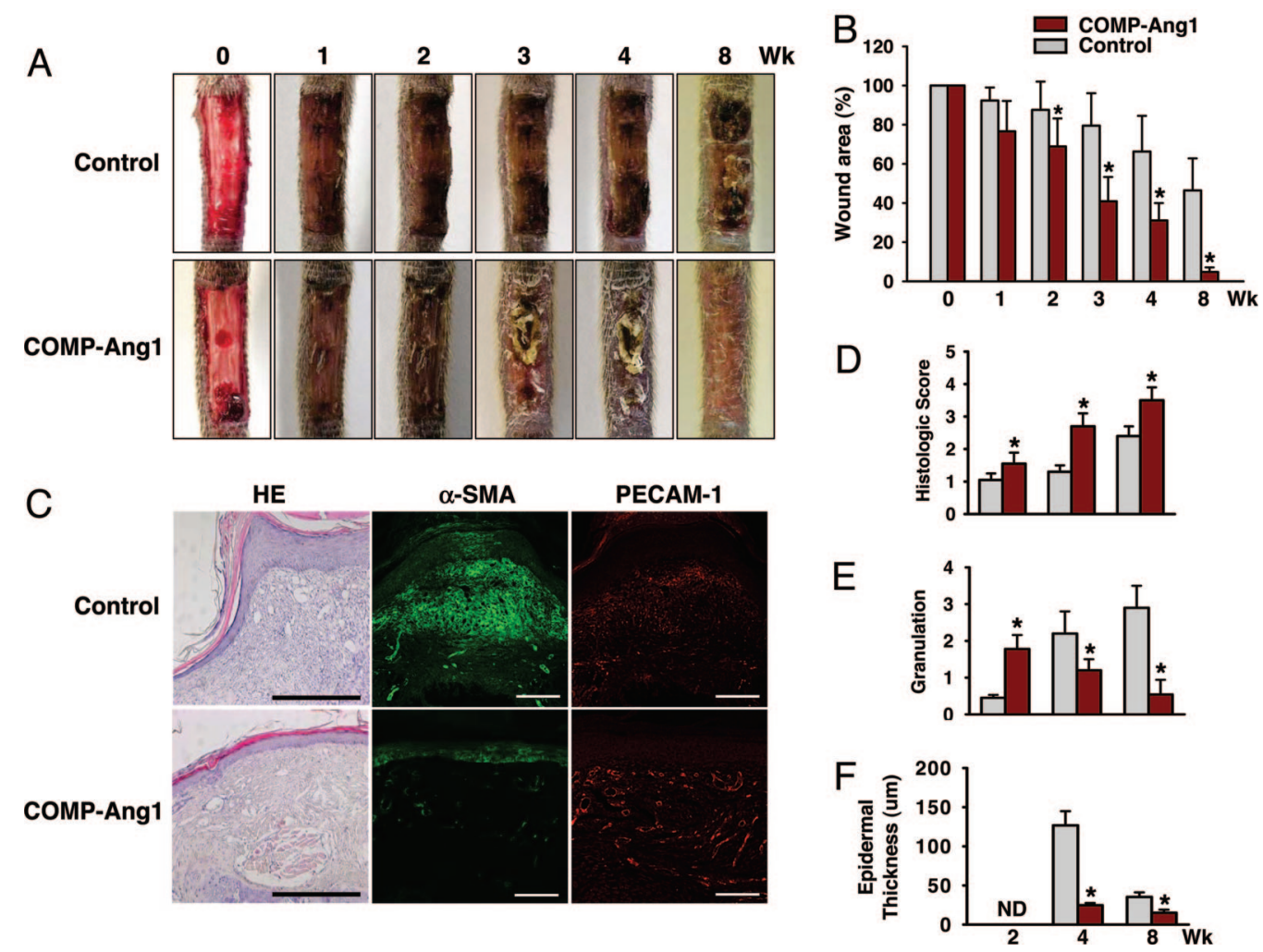

Fig. 2. COMP-Ang1 accelerates wound healing in tail skin of diabetic mice. An excisional full-thickness wound (approximate area, $30 \mathrm{~mm}^{2}$ ) was made in the tail skin of diabetic $d b / d b$ mice, and mice were treated with $1 \times 10^{9}$ pfu of Ade- $\beta$-gal (Control) or Ade-COMP-Ang1 (COMP-Ang1) virus. At the indicated weeks later, tails were photographed $(A)$, wound areas were measured $(B)$, and regenerative activities of epidermis and dermis $(D)$, granulation thickness $(E)$, and epidermal thickness $(F)$ were measured. ND, not determined. (C) Representative photographs of hematoxylin/eosin (HE) staining and $\alpha$-smooth muscle actin $(\alpha$-SMA) (green) and PECAM-1 (red) immunostaining of sections of wound areas of mice treated with COMP-Ang1 and control virus 8 weeks after treatment. (Scale bars, $100 \mu \mathrm{m}$.) All bars shown in $B$ and $D-F$ represent mean \pm SD from five mice. *, $P<0.01$ versus control at each time point.

blood flow rates $(\mathrm{ml} / \mathrm{min}$ per $100 \mathrm{~g}$ of tissue) in wounded regions of COMP-Ang1-treated mice $(n=5)$ were 1.26- to 1.31-fold $(P<$ $0.01)$ and 1.38- to 1.42-fold $(P<0.01)$ greater than control-treated mice $(n=5) 2$ and 4 weeks, respectively, after treatment (Fig. $3 E$ and $F$ ). These results suggest that COMP-Ang1-induced acceleration of wound healing in diabetic mice could be mediated by relief
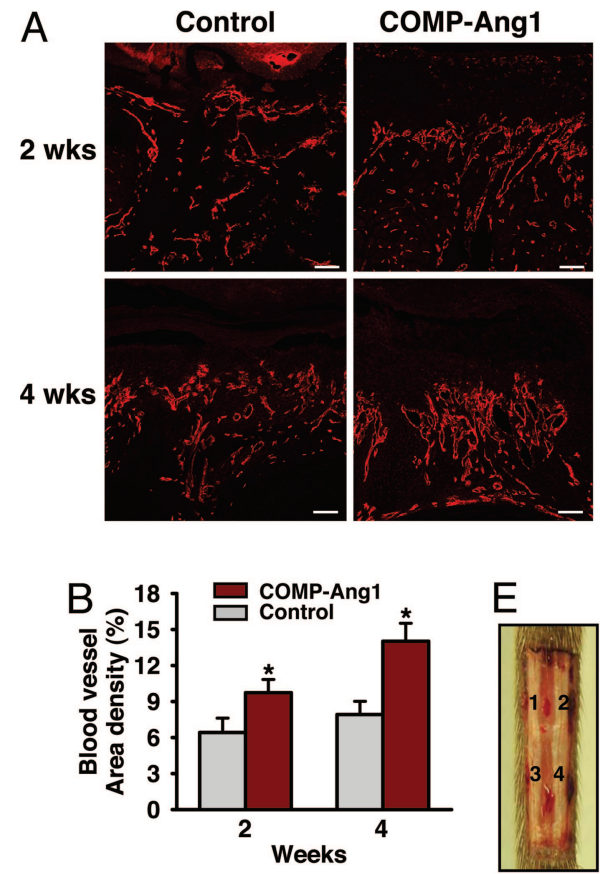
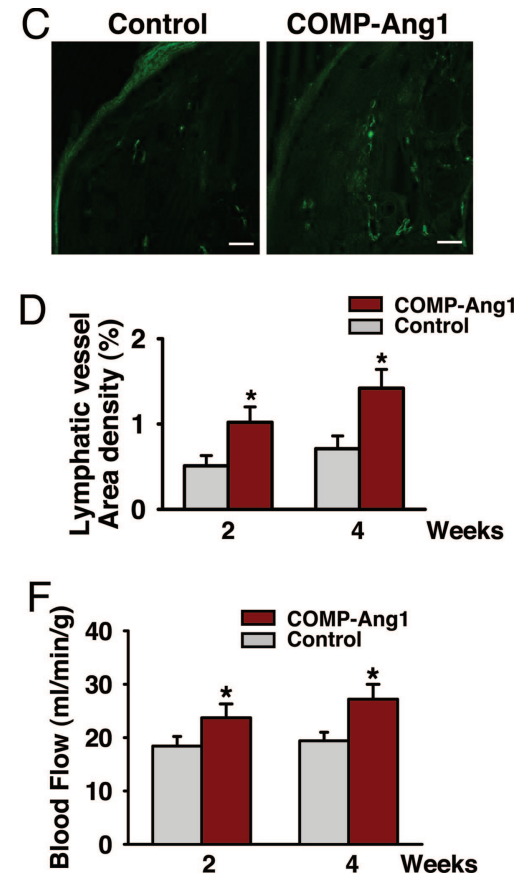

Fig. 3. COMP-Ang1 promotes angiogenesis and blood flow in the wound region of tail skin. An excisional full-thickness wound (approximate area, $30 \mathrm{~mm}^{2}$ ) was made in the tail skin of diabetic $d b / d b$ mice, and mice were treated with $1 \times 10^{9} \mathrm{pfu}$ of Ade- $\beta$-gal (Control) or Ade-COMP-Ang1 (COMP-Ang1) virus. Two $(A)$ and four ( $A$ and $C$ ) weeks later, blood and lymphatic vessels were visualized with PECAM-1 (red) $(A)$ and LYVE-1 (green) (C) immunostaining, and area densities of blood $(B)$ and lymphatic $(D)$ vessels were determined. (Scale bars, 50 $\mu \mathrm{m}$.) Each bar represents mean \pm SD from five mice. $(E)$ By using a laser Doppler flowmeter (Transonic Systems), tissue blood flow in four regions $(1,2,3$, and 4$)$ of the wound area on the dorsal side of the tail were measured. $(F)$ Quantification of skin blood flow at each of the four numbered regions in $E$ was performed, and mean values were obtained 2 and 4 weeks after treatment with control or COMP-Ang1 virus. Each bar represents mean \pm SD from four mice. *, $P<0.05$ versus control at each time point. 

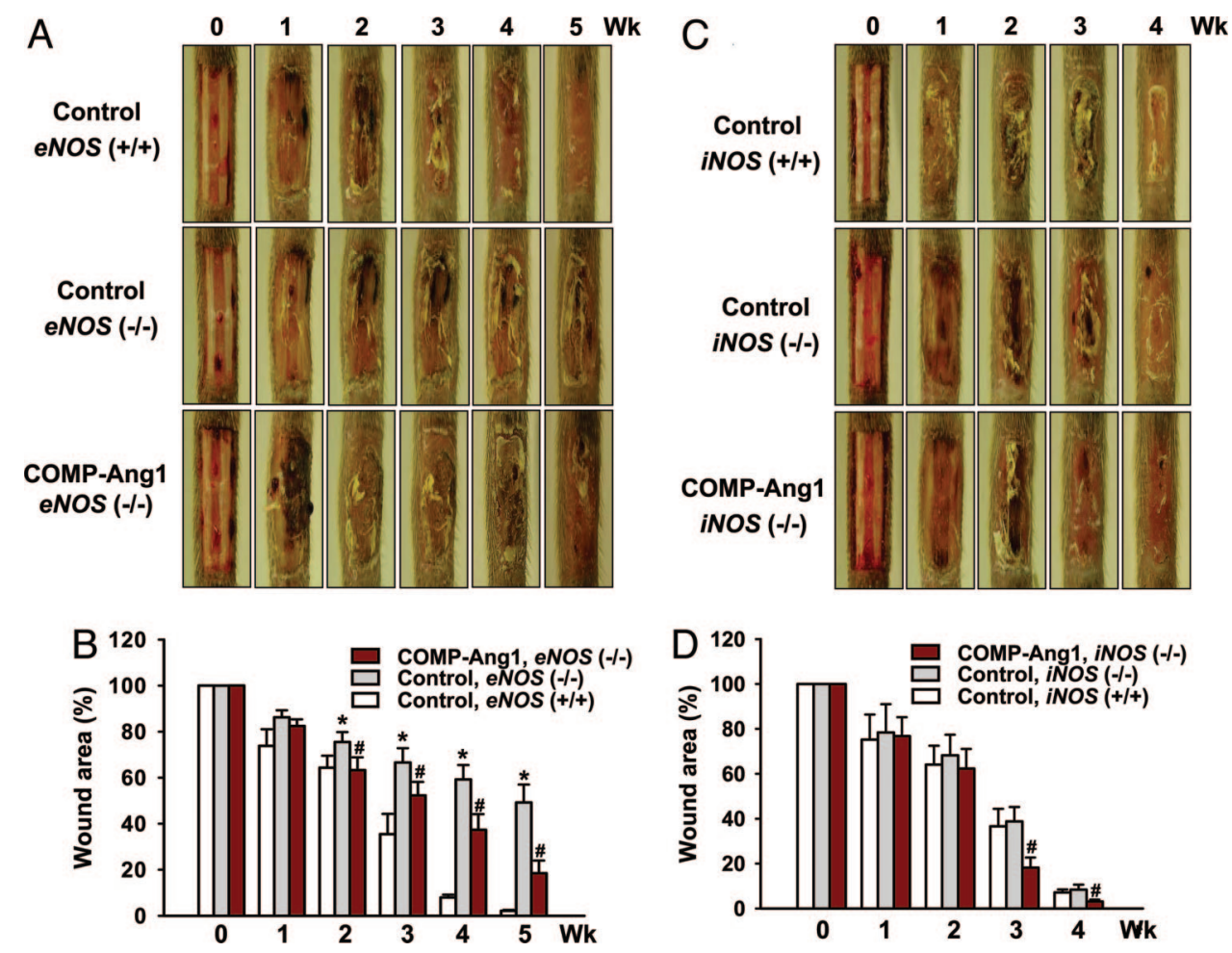

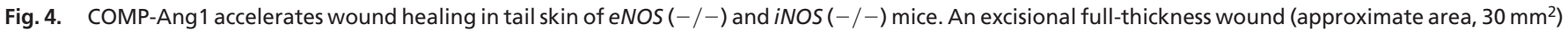

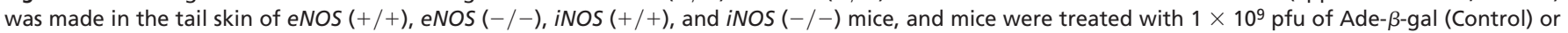

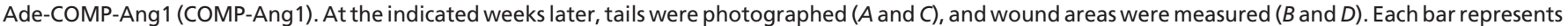

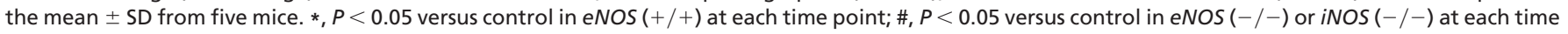
point.

of ischemia in diabetic skin through COMP-Ang1-induced enhanced blood flow. In addition, mice treated with COMP-Ang1 showed higher proliferative activity of the basal cells of epidermis compared with control mice (Fig. 7, which is published as supporting information on the PNAS web site). Because angiogenesis precedes development (25-27) and repair (28) of organs, and blood vessels function as more than carriers of nutrients and passive filters of blood in tissues, our results suggest that COMP-Ang1 may promote skin regeneration by enhancing the proliferation of epidermal basal cells in diabetic wounds, possibly through enhancing angiogenesis.

COMP-Ang1 Accelerates Wound Healing in Tail Skin of eNOS $(-/-)$ and iNOS (-/-) Mice. eNOS-induced nitric oxide plays an integral role in normal wound healing $(29,30)$. We observed that $e N O S(-/-)$ mice displayed impaired wound closure by $\approx 40 \%$ and delayed epidermal and dermal regeneration compared with wild-type mice in the tail-wounding model (Fig. $4 A$ and $B$ and Fig. 8, which is published as supporting information on the PNAS web site), which is consistent with previous findings (31). By contrast, iNOS (-/-) mice did not display delayed wound healing or delayed epidermal and dermal regeneration compared with $i N O S(+/+)$ mice (Figs. $4 C$ and $D$ and 8 ). In addition, Ang1-induced angiogenesis appears to require generation of NO by activated eNOS in the endothelium (22). Therefore, we asked whether eNOS or iNOS participated in COMP-Ang1-induced accelerated wound healing by undertaking tail wounding of 9- to 10-week-old eNOS (-/-) and $i N O S(-/-)$ mice. Time course observations indicated that eNOS $(-/-)$ and $i N O S(-/-)$ mice treated with COMP-Ang1 showed accelerated wound closure and epidermal and dermal regeneration compared with control mice (Figs. 4 and 8). Moreover, eNOS (-/-) and $i N O S(-/-)$ mice treated with COMP-Ang1 showed enhanced angiogenesis compared with mice treated with control virus (data not shown). These findings indicate that enhanced angiogenesis mediated by COMP-Ang1 during wound healing in tail skin does not depend on eNOS or iNOS alone.

Topical COMP-Ang1 Promotes Wound Healing with Enhanced Angiogenesis and Blood Flow in Tail Skin. Given that COMP-Ang1 antagonizes delayed wound healing in diabetic mice, we asked whether topical application of recombinant COMP-Ang1 protein promotes such an effect. To this end, a stable Chinese hamster ovary cell line producing recombinant COMP-Ang1 protein (CA1-2, production level, 20-30 mg/liter) was established (17). To test the efficiency of topical application of COMP-Ang1 protein, we directly applied 50 $\mu \mathrm{l}$ of saline $(0.9 \% \mathrm{NaCl})$ containing $100 \mu \mathrm{g}$ of COMP-Ang1 protein on a daily basis into full-thickness wounds created on the dorsal side of the tail in diabetic C57BLKS/J-m $+/+\operatorname{Lepr}^{d b}(d b / d b)$ mice. In parallel, wounds made in littermates were treated daily with BSA as a control. Applied proteins were mainly distributed at wound edges around the dressing film during the first week and became evenly distributed to the entire wound area during the 3 weeks after treatment (Fig. 9, which is published as supporting information on the PNAS web site). Wounds receiving COMP-Ang1 protein healed faster than wounds receiving BSA. In COMP-Ang1 $(n=6)$ versus BSA $(n=6)$, values for epidermal closure $\left(\mathrm{mm}^{2}\right)$ from the initial wound were $9.5 \mathrm{~mm}^{2}$ versus $3.5 \mathrm{~mm}^{2}$ at 2 weeks, $P<0.01$, and $17.0 \mathrm{~mm}^{2}$ versus $5.5 \mathrm{~mm}^{2}$ at 4 weeks, $P<0.01$ (Fig. $5 A$ and $B$ ), respectively. Likewise, wounds receiving COMP-Ang1 protein displayed accelerated epidermal and dermal regeneration $(\approx 1.4$-fold on day 14 and $\approx 1.9$-fold at 4 weeks) (Fig. $5 C$ ), enhanced angiogenesis $(\approx 1.3$-fold on day 14 and $\approx 1.5$-fold at 4 weeks $)$, and enhanced lymphangiogenesis $(\approx 1.5$-fold on day 14 and $\approx 1$. 7 -fold at 4 weeks) (Fig. $5 D-F)$. Moreover, overall blood flow rates $(\mathrm{ml} / \mathrm{min}$ per $100 \mathrm{~g}$ of tissue) in wounded regions of COMP-Ang1-treated 

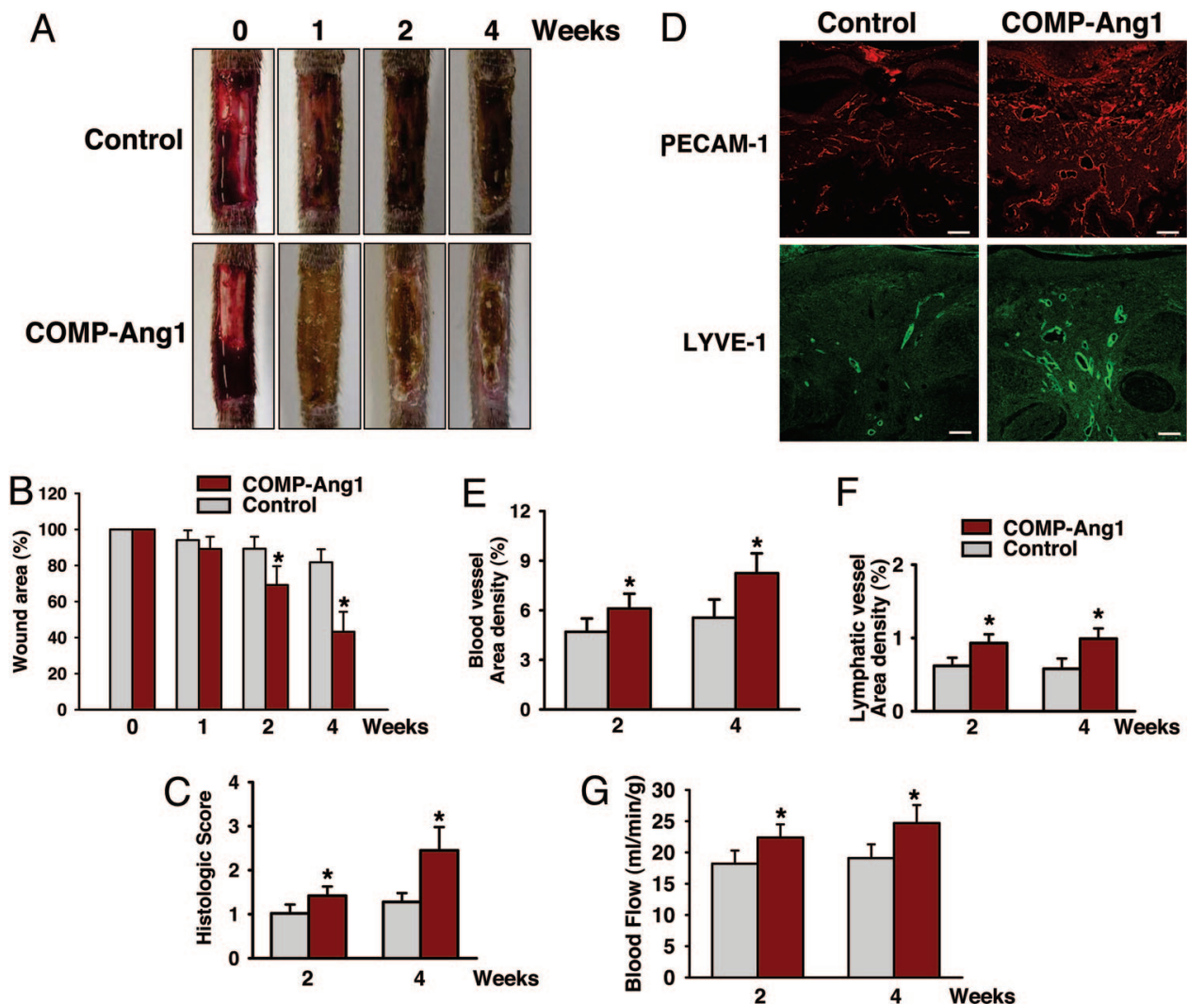

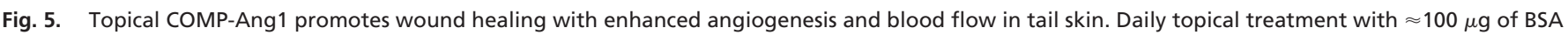

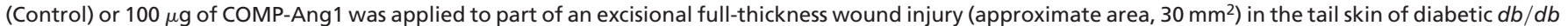

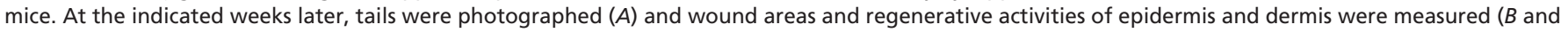

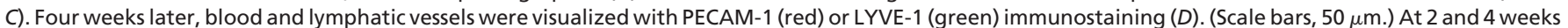

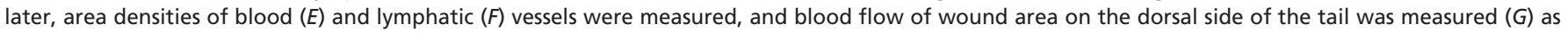
described in the Fig. 3 legend. Each bar represents mean \pm SD from six mice. *, $P<0.05$ versus control at each time point.

mice $(n=5)$ were 1.21 - to 1.25 -fold $(P<0.01)$ and 1.28 - to 1.31 -fold $(P<0.01)$ greater than control-treated mice $(n=5) 2$ and 4 weeks, respectively, after treatment (Fig. 5G). Unlike topical application of human VEGF $(12,13)$, obvious edema formation and vascular leakage were not observed in wound beds during wound healing after administration of COMP-Ang1.

\section{Conclusion}

Systemic and topical COMP-Ang1 accelerates cutaneous wound closure with enhanced angiogenesis and lymphangiogenesis and higher blood flow in normal and diabetic mice, and these effects are not dependent on eNOS or iNOS alone. Ang1 chimeras similar to COMP1-Ang1 could represent new therapeutics to promote cutaneous wound healing in diabetes.

\section{Methods}

Generation of Ade-COMP-Ang1 and COMP-Ang1 Recombinant Protein. Recombinant adenovirus expressing COMP-Ang1 or bacterial $\beta$-gal was constructed by using the pAdEasy vector system (Qbiogene, Carlsbad, CA), as described in ref. 17. Recombinant Chinese hamster ovary cells expressing COMP-Ang1 (CA1-2) were established, and recombinant COMP-Ang1 protein was prepared as described in ref. 17 .

Animals and Treatment. Specific pathogen-free FVB/N, diabetic C57BLKS/J-m + / $\operatorname{Lepr}^{d b}(d b / d b)$, C57BL/6J, and eNOS $(-/-)$ and $i N O S(-/-)$ (C57BL/6J genetic background) mice were purchased from The Jackson Laboratory and bred in our pathogen-free animal facility. Eight- to 10 -week-old male mice were used for this study. Animal care and experimental procedures were performed under approval from the Animal Care Committees of the Korea Advanced Institute of Science and Technology (KAIST) and the Korea Research Institute of Chemical Technology (KRICT). For hole-punch assays, a $1.5-\mathrm{mm}$ hole was made in the center of both ears of $\mathrm{FVB} / \mathrm{N}$ mice by using a metal ear punch (Harvard Apparatus). For fullthickness wounding, excisions were made on the dorsal surface of the tail (23), $\approx 0.5-1.0 \mathrm{~cm}$ distal to the body of the animal. A template was used to mark a $10 \times 3 \mathrm{~mm}$ area on the dorsal surface of the tail. Full-thickness wounds corresponding to the template area were created by using individual sterile \#10 gauge scalpels (Becton Dickinson). Bleeding was stopped by pressure application, and wounds were covered with a film spray dressing (Cavilon; 3M). Postoperatively, mice were kept warm, and their temperature was monitored for 3 days after surgery. To prevent infection, trimethoprim sulfa (Sulfatrim pediatric suspension; Ratiopharm, Ulm, Germany) was added to the drinking water for 5 days. Harvesting of ears and tails for wound-closure analysis required anesthesia by intramuscular injection of a combination of anesthetics ( $80 \mathrm{mg} / \mathrm{kg}$ ketamine and $12 \mathrm{mg} / \mathrm{kg}$ xylazine) during the course of the study from day 0 (immediately after wounding) until 8 weeks after wounding. For adenoviral treatment, $1 \times 10^{9}$ pfu of Ade-COMP-Ang1 or control virus diluted in $50 \mu \mathrm{l}$ of sterile $0.9 \% \mathrm{NaCl}$ was injected intravenously into the tail vein $12 \mathrm{~h}$ after wounding. To detect circulating COMP-Ang1, we used an established ELISA protocol (17). For protein treatment, $100 \mu \mathrm{g}$ of COMP-Ang1 recombinant protein or BSA dissolved in $50 \mu \mathrm{l}$ of sterile $0.9 \% \mathrm{NaCl}$ was directly applied to 
wound sites before the dressing on the first day and applied primarily to exposed marginal wound areas between the film spray dressing and nonwound areas daily for 4 weeks after wounding. To examine the distribution of applied proteins, fluorescein-conjugated COMP-Ang1 was prepared by using a Fluorescein-EX protein labeling kit (Molecular Probes) according to the manufacturer's protocol, and fluorescein-conjugated BSA was purchased (Molecular Probes). Proteins were topically applied to wound sites in the same manner, and their distribution was evaluated by an external excitation fluorescence-equipped dissecting microscope (Stemi SV6; Zeiss).

Morphometric Analysis of Wound Closure. Mice were anesthetized by intramuscular injection with a combination of anesthetics $(80$ $\mathrm{mg} / \mathrm{kg}$ ketamine and $12 \mathrm{mg} / \mathrm{kg}$ xylazine) and placed prone on a warming pad. Wounds were photographed with a digital camera (Coolpix 8400; Nikon). Ear-hole diameters (in $\mathrm{mm}$ ) and tailwound areas (in $\mathrm{mm}^{2}$ ) were calculated from wound perimeter tracings by using photographic analysis in IMAGEJ software (http://rsb.info.nih.gov/ij). Tail-wound areas were interpreted as $100 \%$ on day 0 of the week of wounding; wound areas on subsequent days were expressed as a percentage of the day 0 value.

Histologic and Morphometric Analysis. Mouse tissues were fixed by vascular perfusion of $1 \%$ paraformaldehyde in PBS, the ears and tails of the mice were removed, and ear tissues were wholemounted and tail tissues were embedded in paraffin (for hematoxylin and eosin staining) or cryofreezing medium (for immunostaining). Paraffin sections (6- $\mu \mathrm{m}$ thickness) and cryosections (20- $\mu \mathrm{m}$ thickness) were prepared and incubated for $1 \mathrm{~h}$ at room temperature with blocking solution containing 5\% normal goat serum (Jackson ImmunoResearch) in PBS with $0.3 \%$ Triton X-100 (PBST). Whole-mounted ear or tail sections were incubated for $2 \mathrm{~h}$ at room temperature with one or more of the following primary antibodies: (i) for blood vessels, antiPECAM-1 antibody, hamster clone 2H8, 1:100 (Chemicon International, Temecula, CA); (ii) for lymphatic vessels, antimouse LYVE-1 antibody, rat monoclonal antibody, 1:100 (Aprogen, Daejeon, Korea); (iii) for fibroblasts, FITCconjugated anti- $\alpha$-smooth muscle actin antibody, mouse clone 1A4, 1:200 (Sigma-Aldrich); (iv) for proliferating cells, anti-Ki67 antibody, rabbit polyclonal antibody, 1:100 (NovoCastra, Newcastle, U.K.); (v) and for neural cells, antineurofilament antibody, rabbit polyclonal antibody, 1:100 (Chemicon International). After several washes in PBST, sections were incubated

1. Martin, P. (1997) Science 276, 75-81.

2. Tonnesen, M. G., Feng, X. \& Clark, R. A. (2000) J. Invest. Dermatol. Symp. Proc. 5, 40-46.

3. Hirakawa, S. \& Detmar, M. (2004) J. Dermatol. Sci. 35, 1-8.

4. Yancopoulos, G. D., Davis, S., Gale, N. W., Rudge, J. S., Wiegand, S. J. \& Holash, J. (2000) Nature 407, 242-248.

5. Tammela, T., Petrova, T. V. \& Alitalo, K. (2005) Trends Cell Biol. 15, 434-441.

6. The Diabetes Control and Complications Trial Study Group (1993) N. Engl. J. Med. 329, 977-986.

7. Martin, A., Komada, M. R. \& Sane, D. C. (2003) Med. Res. Rev. 23, 117-145.

8. Laing, P. (1998) Am. J. Surg. 176, 11S-19S.

9. Reiber, G. E., Vileikyte, L., Boyko, E. J., del Aguila, M., Smith, D. G., Lavery, L. A. \& Boulton, A. J. (1999) Diabetes Care 22, 157-162.

10. Frank, S., Hubner, G., Breier, G., Longaker, M. T., Greenhalgh, D. G. \& Werner, S. (1995) J. Biol. Chem. 270, 12607-12613.

11. Kampfer, H., Pfeilschifter, J. \& Frank, S. (2001) Lab. Invest. 81, 361-373.

12. Galeano, M., Deodato, B., Altavilla, D., Cucinotta, D., Arsic, N., Marini, H., Torre, V., Giacca, M. \& Squadrito, F. (2003) Diabetologia 46, 546-555.

13. Galiano, R. D., Tepper, O. M., Pelo, C. R., Bhatt, K. A., Callaghan, M., Bastidas, N., Bunting, S., Steinmetz, H. G. \& Gurtner G. C. (2004) Am. J. Pathol. 164, 1935-1947.

14. Thurston, G., Suri, C., Smith, K., McClain, J., Sato, T. N., Yancopoulos, G. D. \& McDonald, D. M. (1999) Science 286, 2511-2514.

15. Davis, S., Aldrich, T. H., Jones, P. F., Acheson, A., Compton, D. L., Jain, V., Ryan, T. E., Bruno, J., Radziejewski, C., Maisonpierre, P. C., et al. (1996) Cell 87, 1161-1169.

16. Suri, C., Jones, P. F., Patan, S., Bartunkova, S., Maisonpierre, P. C., Davis, S., Sato, T. N. \& Yancopoulos, G. D. (1996) Cell 87, 1171-1180. for $1 \mathrm{~h}$ at room temperature with one or more secondary antibodies: (i) Cy3-conjugated anti-hamster IgG antibody, 1:500 (Jackson ImmunoResearch); (ii) FITC-conjugated anti-rat antibody or anti-rabbit antibody, 1:500 (Jackson ImmunoResearch). For control experiments, the primary antibody was omitted or replaced by preimmune serum. Signals were visualized and digital images were obtained by using a Zeiss Apotome microscope and a Zeiss LSM 510 confocal microscope equipped with argon and helium-neon lasers (Zeiss). The extent of wound healing in the ear $(\mathrm{mm})$ was measured by photographic analysis of immunofluorescent images with image analysis software (LSM IMAGE VIEWER; Zeiss). The parameters of epidermal and dermal regeneration, thickness of tissue granulation, and thickness of epidermis were evaluated by using hematoxylin/eosin-stained sections and scored (Table 1). Area densities (percentage of tissue area) of blood and lymphatic vessels in healing margins of the ear and wounding sections of the tail were measured by PECAM-1- and LYVE-1- immunopositive blood and lymphatic vessels, respectively, at a magnification of $\times 200$ in five regions, each $0.21 \mathrm{~mm}^{2}$ area, per mount or section.

Measurement of Tissue Blood Flow in Wound Areas. Mice were anesthetized and placed on a heated table, and a type $\mathrm{N}$ flowprobe (Transonic Systems) was placed on four regions of the tail wound without pressure, which would occlude vessels and reduce perfusion in the area of interest. The flowprobe was kept in place on the position of the highest sensitivity by a micromanipulator and connected to a laser Doppler flowmeter (model BLF21; Transonic Systems), which can measure microcirculation in $1 \mathrm{~mm}^{3}$ of tissue for real-time assessment of perfusion. These analog signals were digitized at $100 \mathrm{~Hz}$ (Digidata 1200; Axon Instruments, Foster City, CA) and continuously displayed by a data acquisition program. The mean tissue perfusion rate $(\mathrm{ml} /$ min per $100 \mathrm{~g}$ of tissue) was analyzed by using AXOSCOPE 9.0 software (Axon Instruments).

Statistics. Values presented are mean \pm SD. Significant differences between means were determined by analysis of variance followed by the Student-Newman-Keul's test. Statistical significance was set at $P<0.05$ or $P<0.01$.

We thank Ki-Nam Min and Jin Sun Kwak for technical assistance. This work was supported in part by National Research Laboratory Program Grant 2004-02376 (to G.Y.K.) and Nano-Bio Research and Development Program Grant 2004-02318 (to H.J.H.) of the Korean Ministry of Science and Technology, Bio-Challenge Program Grant M1-0310-000042 (to G.Y.K.), and Korea Health R\&D Project Grant 0405-DB010104-0006 (to G.Y.K.) of the Ministry of Health and Welfare.
17. Cho, C. H., Kim, K. E., Byun, J., Jang, H. S., Kim, D. K., Baluk, P., Baffert, F., Lee, G. M. Mochizuki, N., Kim, J., et al. (2005) Circ. Res. 97, 86-94.

18. Saharinen, P., Kerkela, K., Ekman, N., Marron, M., Brindle, N., Lee, G. M., Augustin, H Koh, G. Y. \& Alitalo, K. (2005) J. Cell Biol. 169, 239-243.

19. Carlson, T. R., Feng, Y., Maisonpierre, P. C., Mrksich, M. \& Morla, A. O. (2001) J. Biol. Chem. 276, 26516-26525.

20. Dallabrida, S. M., Ismail, N., Oberle, J. R., Himes, B. E. \& Rupnick, M. A. (2005) Circ. Res. 96, e8-e24.

21. Cho, C. H., Kammerer, R. A., Lee, H. J., Steinmetz, M. O., Ryu, Y. S., Lee, S. H., Yasunaga, K., Kim, K. T., Kim, I., Choi, H. H., et al. (2004) Proc. Natl. Acad. Sci. USA 101, 5547-5552. 22. Babaei, S., Teichert-Kuliszewska, K., Zhang, Q., Jones, N., Dumont, D. J. \& Stewart, D. J. (2003) Am. J. Pathol. 162, 1927-1936.

23. Falanga, V., Schrayer, D., Cha, J., Butmarc, J., Carson, P., Roberts, A. B. \& Kim, S. J. (2004) Wound Repair Regen. 12, 320-326.

24. Coleman, D. L. (1982) Diabetes 31, 1-6.

25. Matsumoto, K., Yoshitomi, H., Rossant, J. \& Zaret, K. S. (2001) Science 294, 559-563.

26. Cleaver, O. \& Melton, D. A. (2003) Nat. Med. 9, 661-668.

27. Fukumura, D., Ushiyama, A., Duda, D. G., Xu, L., Tam, J., Krishna, V., Chatterjee, K. Garkavtsev, I. \& Jain, R. K. (2003) Circ. Res. 93, e88-e97.

28. Franck-Lissbrant, I., Haggstrom, S., Damber, J. E. \& Bergh, A. (1998) Endocrinology 139, $451-456$.

29. Witte, M. B. \& Barbul, A. (2002) Am. J. Surg. 183, 406-412.

30. Schwentker, A., Vodovotz, Y., Weller, R. \& Billiar, T. R. (2002) Nitric Oxide 7, 1-10.

31. Lee, P. C., Salyapongse, A. N., Bragdon, G. A., Shears, L. L., II, Watkins, S. C., Edington, H. D. \& Billiar, T. R. (1999) Am. J. Physiol. 277, H1600-H1608. 